\title{
An Investigation of Electronic Structure and Aromaticity in Medium-Sized Nanoclusters of Gold-Doped Germanium
}

\author{
Xiao-Jun Li, ${ }^{1,2}$ Hong-Jiang Ren, ${ }^{2}$ and Li-Ming Yang ${ }^{3}$ \\ ${ }^{1}$ School of Natural and Applied Sciences, Northwestern Polytechnical University, Shaanxi, Xi'an 710072, China \\ ${ }^{2}$ School of Chemistry and Chemical Engineering, Xi'an University of Arts and Science, Shaanxi, Xi'an 710072, China \\ ${ }^{3}$ Center for Theoretical and Computational Chemistry (CTCC), Department of Chemistry, University of Oslo (UiO), \\ P.O. Box 1033, Blindern, 0315 Oslo, Norway
}

Correspondence should be addressed to Xiao-Jun Li, lixiaojun0@yahoo.com.cn and Li-Ming Yang, lmyang.uio@gmail.com

Received 19 October 2012; Accepted 3 November 2012

Academic Editor: Su Chen

Copyright (๑) 2012 Xiao-Jun Li et al. This is an open access article distributed under the Creative Commons Attribution License, which permits unrestricted use, distribution, and reproduction in any medium, provided the original work is properly cited.

The electronic property and aromaticity of endohedrally doped $\mathrm{AuGe}_{10}{ }^{-}$and $\mathrm{AuGe}_{12}{ }^{-}$clusters are investigated using the density-functional theory (DFT) within the hybrid B3LYP method. The calculated results reveal that the two clusters have high thermodynamic stability reflected by reaction energy. At the same time, it could be hoped that their high stability may arise from the closed-shell spherical aromaticity with eight $\pi$-electrons satisfying the $2\left(N_{n}+1\right)^{2}$ counting rule with $N_{\pi}=1$. A popular nucleusindependent chemical shifts (NICSs) calculation on basis of magnetic shieldings is also performed to confirm the aromaticity of the three-dimensional nanoclusters with largely negative NICS values. In addition, the electronic features and chemical bonding of the two clusters are analyzed with the help of the density of states (DOS) and electron localization function (ELF), and the majority of Ge-Ge bonds on the cage show more covalent characters.

\section{Introduction}

Semiconductor clusters, especially germanium, have been extensively investigated both experimentally and theoretically due to their potential applications in the microelectronics industry [1-6] and are of considerable scientific interest to explore the chemical structure and bonding as well as their thermodynamic stability and photoelectric effect. Unlike silicon, the effective masses of electron and hole in germanium are light; it can generate high carrier mobility and makes germanium attractive for high-performance field-effect logic transistors [7]. However, pure germanium clusters with medium or large size are chemically unstable and quite reactive because of the existence of abundant dangling bonds [5]. Interestingly, the encapsulation of different transitionmetal (TM) atoms [8-10] in the medium-sized clusters can saturate the dangling bonds on the germanium cage surface and form $s p^{3}$ hybridization, and thereafter stabilize the germanium cages. Consequently, the metal-encapsulated germanium clusters exhibit many fascinating features such as nanostructures, for example, thermodynamic stability [11,
12], magnetic and superconducting behaviors [ $8,13,14]$, photoelectric effect $[15,16]$, and reduced energy gap between the highest occupied molecular orbital (HOMO) and the lowest unoccupied molecular orbital (LUMO) $[9,17,18]$. Some metal-encapsulated Ge clusters were also proposed as novel cluster-assembled materials due to the small HOMOLUMO gap within about $2 \mathrm{eV}$, for example, $\mathrm{AuGe}_{n}(n=2-$ 13) [18] and TM@Ge $e_{12}(\mathrm{TM}=\mathrm{Zn}, \mathrm{W}$, and Os) $[19,20]$. In an experimental study, the bimetallic $\mathrm{CoGe}_{10}{ }^{-}$cluster in the mass spectrum was measured using laser vaporization technique [21], and its molecular structure was determined by the density-functional theory (DFT) calculations [22].

To our knowledge, there have been a number of interesting findings on the calculation of metal-encapsulated Ge clusters, for example, $\mathrm{NiGe}_{n}(n=1-13)$ [23], $\mathrm{CrGe}_{n}$ $(n=1-13)$ [24], $\mathrm{WGe}_{n}(n=1-17)$ [25], $\mathrm{TMGe}_{n}(\mathrm{TM}=$ $\mathrm{Ti}, \mathrm{Zr}$, Hf and $\mathrm{Cu} ; n=1-20)[26,27]$, and so on. A few outstanding predictions are medium-sized fullerene-like with 10 or 12 atoms. Kumar et al. [19, 28] reported a series of systematic investigations of TM@ $X_{n}(\mathrm{X}=\mathrm{Si}, \mathrm{Ge}, \mathrm{Sn}$, and $\mathrm{Pb} ; \mathrm{TM}=\mathrm{Ni}, \mathrm{Pd}, \mathrm{Pt}, \mathrm{Zn}$, and $\mathrm{Cd} ; n=10$ and 12 ) 
clusters using an ab initio ultrasoft pseudopotential method and generalized gradient approximation, and these clusters can be further stabilized by doping of an $\mathrm{Ni}$ or $\mathrm{Pt}$ atom, leading to some of the smallest metal-encapsulated clusters. King and coworkers $[29,30]$ explored the possible structures of $\mathrm{M} @ \mathrm{Ge}_{10}{ }^{z}$ systems $(\mathrm{M}=\mathrm{Co}$ and $\mathrm{Be} ; z=-5$ to +2$)$ at the DFT-B3LYP/6-31G(d) level of theory and found that a singlet $D_{5 h}$ pentagonal prismatic structure is the global minimum for the trianionic Co@ $\mathrm{Ge}_{10}{ }^{3-}$ cluster, which is in accordance with the experimental result. Tang et al. [31] investigated the structure, stability, and optical property of $\mathrm{TM} \mathrm{Ge}_{12}(\mathrm{TM}=\mathrm{Sc}-\mathrm{Ni})$ using the relativistic all-electron density functional theory. It was found that all the TM@Ge clusters are perhaps partial metallic, and the ground-state geometry of them is probably pseudoicosahedron. Furthermore, eight $\pi$-electron natures make closed-shell spherical systems aromatic according to the $2\left(N_{\pi}+1\right)^{2}\left[N_{\pi}=1\right]$ rule proposed by Hirsch et al. [32]. In 2005, Chen and coworkers [33] also reported the nucleus-independent chemical shifts (NICSs) approach as an aromaticity criterion based on magnetic properties, which have been applied to characterize the all-metal aromatic/antiaromatic clusters [34].

In this paper, we report an investigation of electronic structure and aromaticity of the $\mathrm{AuGe}_{10}{ }^{-}$and $\mathrm{AuGe}_{12}{ }^{-}$ nanoclusters using the hybrid DFT-B3LYP functional in conjunction with LanL2DZ basis set. The choice of endohedral gold atom is due to the fact that the bimetallic gold clusters have some fascinating physical and chemical properties and were experimentally observed using photoelectron spectroscopy [16, 35-37].

\section{Theoretical Methods}

The molecular structure, electronic property, and spherical aromaticity of $\mathrm{AuGe}_{10}{ }^{-}$and $\mathrm{AuGe}_{12}{ }^{-}$clusters were carried out using the hybrid DFT-B3LYP functional [38, 39] implemented in the Gaussian 09 package [40]. The standard double- $\xi$ LanL2DZ basis set [41-43], which provides effective core potentials (ECPs) to consider the relativistic effects of transition metal atoms, was utilized here. Our previous calculations [18] also confirmed the credibility of the theoretical level, and the deviations within $1-6 \%$ are acceptable. To search for the most stable structures, a great number of structural isomers were considered for each size, on basis of a global search published previously [18, 44], and the local minima of all the stationary point geometries were determined by vibrational frequency analysis. The density of states (DOS) of the most stable $\mathrm{AuGe}_{10}{ }^{-}$and $\mathrm{AuGe}_{12}{ }^{-}$clusters was constructed using GaussSum 2.2 program [45] with the full width at half maximum (FWHM) of $0.3 \mathrm{eV}$, and the molecular orbitals were plotted with the isodensity surfaces $\left(0.02 \mathrm{e} / \mathrm{a} . \mathrm{u}^{3}{ }^{3}\right)$. In addition, the nucleus-independent chemical shifts (NICS) were calculated by gauge-independent atomic orbital (GIAO) method $[46,47]$ at the B3LYP/LanL2DZ level of theory. In order to obtain more accurate NICS values, the correlation-consistent cc-pVTZ basis set [48] was also used for Ge atom, whereas the energy-adjusted small-core (19 valence electrons) pseudopotentials (PPs) [49] were used for $\mathrm{Au}$ atom, labeled as cc-pVTZ-PP, and the single-point GIAO calculations at the B3LYP/cc-pVTZ-PP level of theory were performed based on the B3LYP/LanL2DZ-optimized geometries.

\section{Results and Discussion}

3.1. Geometry and Stability. Using the computation scheme described above, we have extensively explored a number of initial isomers formed from taking the previously reported structures of different $\mathrm{TMGe}_{10}$ systems, or doping one $\mathrm{Au}$ dopant at all possible positions of the lower-lying $\mathrm{Ge}_{10}$ isomers, or replacing one $\mathrm{Ge}$ atom of the lower-lying $\mathrm{Ge}_{11}$ isomers with an $\mathrm{Au}$ atom to form new isomers. As a result, the most stable $\mathrm{AuGe}_{10}{ }^{-}$cluster is found to be an endohedrally Au-doped pentagonal prism (see Figure 1(a)). It is worth mentioning that, when an electron is added to the lowest-energy neutral $\mathrm{AuGe}_{10}$ cluster in $C_{2 v}$ symmetry [18], the high $D_{5 h}$-symmetric anionic $A u G e_{10}{ }^{-}$cluster with a ${ }^{1} \mathrm{~A}_{1}{ }^{\prime}$ electronic state forms due to the equal electron distribution for each $\mathrm{Ge}$ atom [44], indicating that the neutral $\mathrm{AuGe}_{10}$ cluster is an electron-deficient base. The geometry of the lowest-energy $\mathrm{AuGe}_{10}{ }^{-}$cluster in $D_{5 h}$ symmetry is also consistent with the neutral $\mathrm{FeGe}_{10}$ cluster [9] as well as the trianionic Co@ $\mathrm{Ge}_{10}{ }^{3-}$ cluster [29]. In the structure, there are 10 equivalent Ge-Ge bond lengths on two five-numbered germanium rings, and 5 equivalent $\mathrm{Ge}-\mathrm{Ge}$ bond lengths on side-jointed prism. At the B3LYP/LanL2DZ level of theory, the equilibrium Ge-Ge bond lengths of them are predicted to be 2.722 , and $2.858 \AA$, respectively; and the equilibrium $\mathrm{Au}-\mathrm{Ge}$ bond lengths in the pentagonal prism are calculated to be $2.721 \AA$, as shown in Table 1 .

Analogous to the formation of $\mathrm{AuGe}_{10}{ }^{-}$cluster, all the possible isomers of $\mathrm{AuGe}_{12}{ }^{-}$cluster are considered. An Au-doped bicapped pentagonal prism is optimized to be the lowest-energy $\mathrm{AuGe}_{12}{ }^{-}$structure, which displays a ${ }^{1} \mathrm{~A}_{1}$ electronic state in $D_{2 d}$ symmetry (see Figure $1(\mathrm{~b})$ ). By comparison, the icosahedral structure of $\mathrm{ZnGe}_{12}$ [50] cluster is confirmed to be the global minima in the potential energy surface (PES), but the icosahedral $\mathrm{AuGe}_{12}{ }^{-}$structure is only a local minimum which is higher in energy than the lowestenergy structure by $0.32 \mathrm{eV}$ [44]. In the bicapped pentagonal prism, the $D_{2 d}$ structure has two $C_{2}$ axes and two $\sigma_{d}$ planes. As a result, four different $\mathrm{Ge}-\mathrm{Ge}$ bond lengths are predicted to be $2.611,2.644,2.647$ and $2.855 \AA$, respectively, whereas the two equilibrium $\mathrm{Au}-\mathrm{Ge}$ bond lengths are calculated to be 2.814 and $2.942 \AA$.

As discussed above, the endohedrally doped $\mathrm{AuGe}_{10}{ }^{-}$ and $\mathrm{AuGe}_{12}{ }^{-}$clusters are the most stable. Obviously, they do not obey the 18-electron counting rule (also known as the octet rule) [51] which may give rise to the high stability of cluster. In 2003, Sen and Mitas [52] also reported the limitation of the 18-electron rule and found that the filling of electron shell according to 18-electron rule is not the only factor in determining the structural stability, and the stability should depend on structural geometry, choice of metal atom, neutral or charged species, and so forth. In addition, one needs to calculate reaction energy to explain 


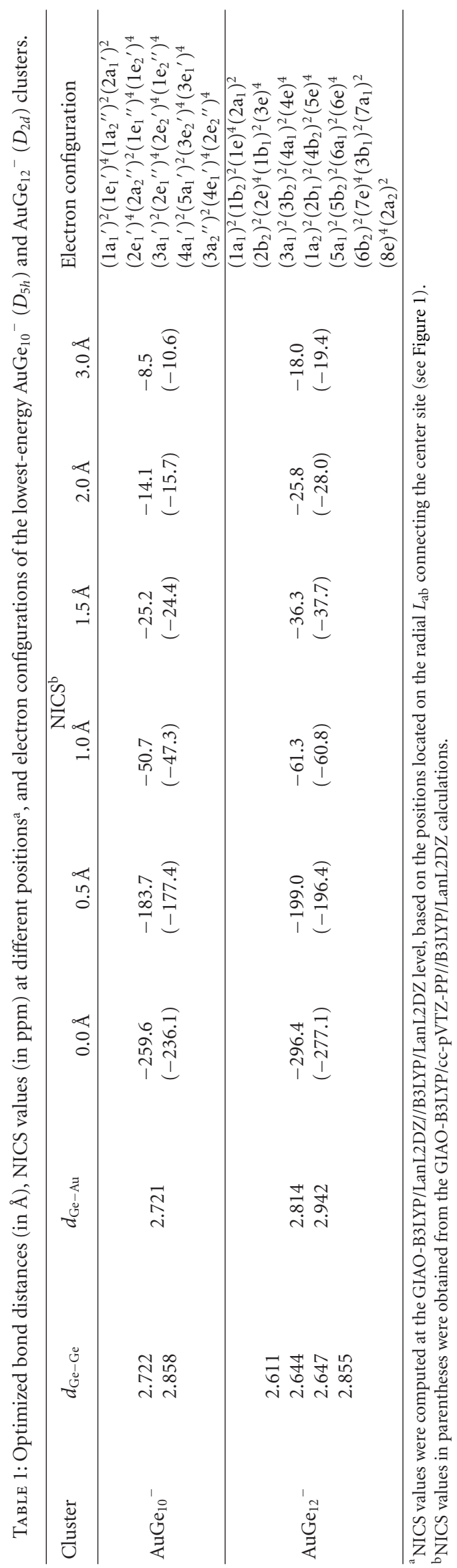




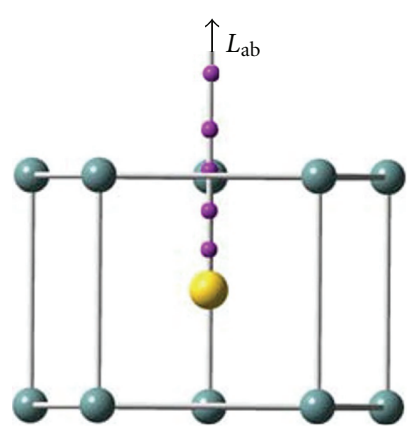

(a)

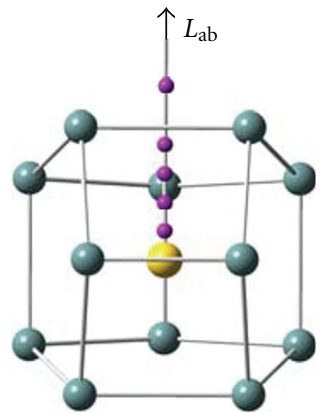

(b)

Figure 1: Equilibrium structures of the lowest-energy $D_{5 h}$-symmetric $A u G_{10}{ }^{-}$(a) and $D_{2 d}$-symmetric AuGe ${ }_{12}^{-}$(b) clusters in [44]. The different NICS sites (purple balls) are indicated.

the thermodynamic stability of the cluster, and the reaction energy $\left(E_{r}\right)$ can be computed according to the following equation:

$$
\begin{aligned}
& E_{r}\left(\mathrm{AuGe}_{n}{ }^{-}\right) \\
& \quad=\frac{\left[E\left(\mathrm{AuGe}_{n}{ }^{-}\right)-n E(\mathrm{Ge})-E\left(\mathrm{Au}^{-}\right)\right]}{(n+1)}, \quad n=10 \text { or } 12,
\end{aligned}
$$

where $E\left(\mathrm{AuGe}_{n}{ }^{-}\right), E(\mathrm{Ge})$, and $E\left(\mathrm{Au}^{-}\right)$represent the total energies of $\mathrm{AuGe}_{n}{ }^{-}, \mathrm{Ge}$, and $\mathrm{Au}^{-}$atoms, respectively. All the total energies include the correction of zero-point vibrational energy (ZPVE). According to our knowledge, the formations of $\mathrm{AuGe}_{10}{ }^{-}$and $\mathrm{AuGe}_{12}{ }^{-}$clusters are exothermic with the large negative $E_{r}$ values, being -2.44 and $-2.50 \mathrm{eV}$. Interestingly, their high thermodynamic stabilities are mainly associated with the geometry and the number of dangling bonds in the two clusters, as mentioned in previous study [18]. Our results also reveal that both of them can appear as stable species, and the two clusters could be synthesized through the exothermic reaction pathway.

3.2. Electronic Structure and Chemical Bonding. In order to explore the electronic features related to the stability of the clusters, we have performed a detailed analysis of the molecular orbitals by examining the partial density of states (PDOS) from the contribution of different orbital components (Au: $s, p, d$; Ge: $s, p$ ) and the electron density of the HOMO and LUMO states. The plots of PDOS of the $\mathrm{AuGe}_{10}{ }^{-}$and $\mathrm{AuGe}_{12}{ }^{-}$clusters are shown in Figures 2(a) and 2(b), respectively.

As for a typical case, each $\mathrm{Ge}$ atom is expected to contribute its four valence electrons to the electron shell configuration of the molecular systems. The $\mathrm{AuGe}_{10}{ }^{-}$cluster with $D_{5 h}$ symmetry thus contains 42 valence electrons which are distributed in the following orbital configuration: $\left(1 \mathrm{e}_{1}{ }^{\prime \prime}\right)^{2}\left(1 \mathrm{e}_{2}{ }^{\prime}\right)^{4}\left(3 \mathrm{a}_{1}{ }^{\prime}\right)^{2}\left(2 \mathrm{e}_{1}{ }^{\prime \prime}\right)^{4}\left(2 \mathrm{e}_{2}{ }^{\prime}\right)^{4}\left(1 \mathrm{e}_{2}{ }^{\prime \prime}\right)^{4}\left(4 \mathrm{a}_{1}{ }^{\prime}\right)^{2}\left(5 \mathrm{a}_{1}{ }^{\prime}\right)^{2}$ $\left(3 \mathrm{e}_{2}{ }^{\prime}\right)^{4}\left(3 \mathrm{e}_{1}{ }^{\prime}\right)^{4}\left(3 \mathrm{a}_{2}{ }^{\prime \prime}\right)^{2}\left(4 \mathrm{e}_{1}{ }^{\prime}\right)^{4}\left(2 \mathrm{e}_{2}{ }^{\prime \prime}\right)^{4}$, as listed in Table 1. Due to the high $D_{5 h}$ symmetry, additionally, the molecular orbitals strongly resemble the spherical features. From the Figure 2(a), we clearly see that the electronic states at high-energy region of around $-3.9 \mathrm{eV}$ above come mainly from $p$-Ge state; and the contribution from $s$ Ge state is very little. The difference is that the valence molecular orbitals at around $-6.0 \mathrm{eV}$ below mainly ascribe to the contribution of $d$-Au and $s$-Ge states; in particular, the $\mathrm{E}_{2}{ }^{\prime}$-type valence orbital at $-6.77 \mathrm{eV}$, holding a doubly degenerate state, is composed of $d$-Au state mixed with $s$-Ge state. Similar behavior is observed for the $D_{2 d}$-symmetric $\mathrm{AuGe}_{12}{ }^{-}$cluster which contains 50 valence electrons with an orbital configuration of $\left[(3 \mathrm{e})^{4}\left(3 \mathrm{a}_{1}\right)^{2}\left(3 \mathrm{~b}_{2}\right)^{2}\left(4 \mathrm{a}_{1}\right)^{2}(4 \mathrm{e})^{4}\left(1 \mathrm{a}_{2}\right)^{2}\left(2 \mathrm{~b}_{1}\right)^{2}\left(4 \mathrm{~b}_{2}\right)^{2}(5 \mathrm{e})^{4}\left(5 \mathrm{a}_{1}\right)^{2}\right.$ $\left.\left(5 \mathrm{~b}_{2}\right)^{2}\left(6 \mathrm{a}_{1}\right)^{2}(6 \mathrm{e})^{4}\left(6 \mathrm{~b}_{2}\right)^{2}(7 \mathrm{e})^{4}\left(3 \mathrm{~b}_{1}\right)^{2}\left(7 \mathrm{a}_{1}\right)^{2}(8 \mathrm{e})^{4}\left(2 \mathrm{a}_{2}\right)^{2}\right]$. The electron density $\left(0.02\right.$ e/a.u. $\left.{ }^{3}\right)$ of HOMO and LUMO states of the two clusters is represented in Figure 2. One can see that both the HOMO and LUMO states are mainly localized around germanium cage, while some electronic distributions around $\mathrm{Au}$ atom are also found in the LUMO. It is remarkable that the encapsulation of the Au dopant is responsible for the geometric rearrangement of pure germanium clusters and the reduction of the HOMOLUMO gaps of pure $\mathrm{Ge}_{10}$ and $\mathrm{Ge}_{12}$ clusters. Especially for the $\mathrm{AuGe}_{12}{ }^{-}$cluster the HOMO and LUMO are nondegenerate and are slightly separated from the adjacent occupied and unoccupied orbitals, resulting in its quite small HOMOLUMO gap of $1.63 \mathrm{eV}$. Thus, although the germanium is semiconductor element, the $\mathrm{AuGe}_{10}{ }^{-}$and $\mathrm{AuGe}_{12}{ }^{-}$clusters may be considered as novel cluster-assembled materials with partially metallic features $[19,44]$.

In 1994, Silvi and Savin [53] reported that the electron localization function (ELF) is currently used to describe the nature of bonding. According to their descriptions, the molecular space is divided into regions or basins of localized electron pairs or attractors. Typically, the existence of a high ELF isovalue (around 0.7 and above) in the bonding region between two atomic basins signifies a localized chemical bond in the region $[44,54]$. For the $D_{5 h}$-symmetric $A u G e_{10}{ }^{-}$ cluster, the ELF basins along Ge-Ge bond upon two fivenumber germanium rings merge at an isovalue of 0.71 (Figure 3(a)), revealing that these Ge-Ge bonds are covalent in nature, while the five Ge-Ge prismy bonds have more metallic or less covalent character reflected by the slightly low ELF isovalue at 0.60 (Figure 3(b)). The ELF contour at a low isovalue of 0.32 (Figure $3(\mathrm{c})$ ) shows two polarized basins 


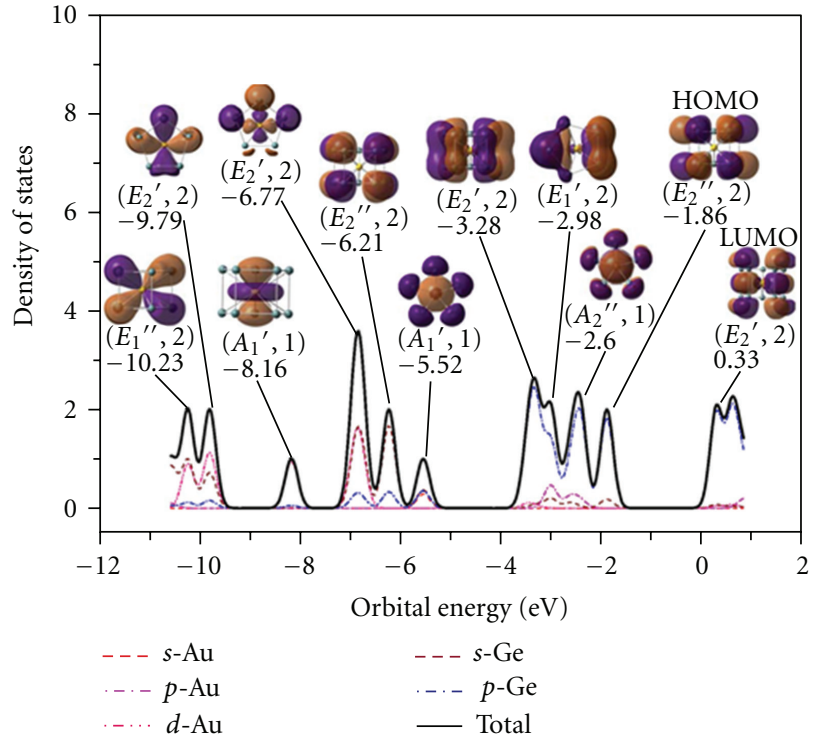

(a)

Figure 2: Density of states (DOS) of the lowest-energy $\mathrm{AuGe}_{10}{ }^{-}$ theory.

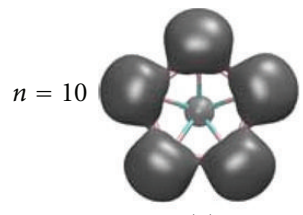

(a)

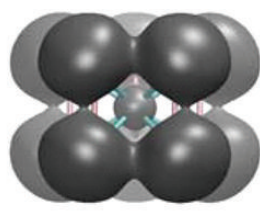

(b)

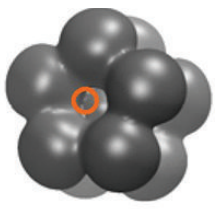

(c)

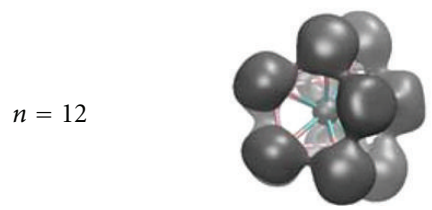

(d)

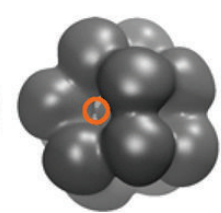

(e)

Figure 3: The ELF isosurfaces of the $\mathrm{AuGe}_{10}{ }^{-}$and $\mathrm{AuGe}_{12}{ }^{-}$clusters. Some different isovalues are indicated: (a) 0.71, (b) 0.60, (c) 0.32, (d) 0.72 , and (e) 0.29 .

between $\mathrm{Au}$ and $\mathrm{Ge}$ atoms, so all the $\mathrm{Au}-\mathrm{Ge}$ bonds are far from being covalent in nature but show an ionic character. The most stable $\mathrm{AuGe}_{12}{ }^{-}$cluster has a high (almost spherical) $D_{2 d}$ symmetry. The covalent bonding pattern between germanium atoms becomes more preferable because all atomic basins along the surface bonds in ELF contours almost merge at an isovalue of 0.72 (Figure 3(d)), whereas the $\mathrm{Au}-\mathrm{Ge}$ bonds display a slightly increased ionic character confirmed by lower ELF isovalue (0.29, Figure 3(e)) and elongated $\mathrm{Au}-\mathrm{Ge}$ bond lengths $(0.093-0.221 \AA)$, as discussed above.

3.3. The $2(N+1)^{2}$ Rule. Aromaticity is well known as one of the important measures of compounds. Compared to

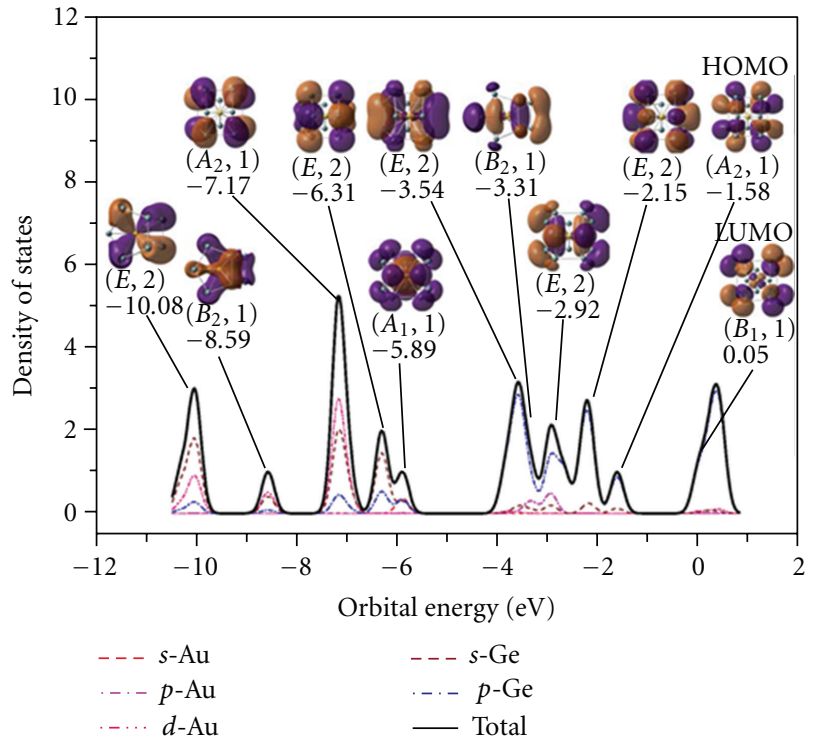

(b)

(a) and $\mathrm{AuGe}_{12}{ }^{-}$(b) clusters computed at the B3LYP/LanL2DZ level of

nonaromatic compounds, analogous aromatic compounds typically give enhanced chemical stability. In general, aromaticity of planar structure can be qualitatively explored using the $4 N+2$ Hückel rule [34]. Another electron counting rule, namely, $2(N+1)^{2}$ rule proposed by Hirsch et al. [32], is proven as an effective aromaticity criterion for spherical species ( $I_{h}$ symmetry), as well as the extension of the treatment to inorganic cage molecules [55]. In the latter electron rule, the $\pi$-electron system of the species can be approximately considered as a spherical electron gas, which surrounds the surface of a sphere [56]. According to the Pauli principle, if the number of $\pi$ electrons in a spherical structure satisfies the $2(N+1)^{2}$ rule, the structure can show a spherical charge distribution and is therefore expected to be aromatic.

The molecular orbitals (MOs) of the $\mathrm{AuGe}_{10}{ }^{-}$and $\mathrm{AuGe}_{12}{ }^{-}$clusters are depicted in Figures 2(a) and 2(b), respectively. We see that the valence electron orbitals of them are divided into two different subsets occupied by $\sigma$ or $\pi$ electrons. In Figure 2(a), the $D_{5 h}$-symmetric $\mathrm{AuGe}_{10}{ }^{-}$cluster contains eight valence $\pi$-electrons in four MOs, for example, $-5.52\left(\mathrm{~A}_{1}{ }^{\prime}, 1\right),-2.98\left(\mathrm{E}_{1}{ }^{\prime}, 2\right)$, and $-2.60\left(\mathrm{~A}_{2}{ }^{\prime \prime}, 1\right)$, and these $\pi$-electrons satisfy the $2\left(N_{\pi}+1\right)^{2}\left[N_{\pi}=1\right]$ counting rule. Similarly, eight valence $\pi$-electrons are also found for the $D_{2 d}$-symmetric $\mathrm{AuGe}_{12}{ }^{-}$cluster (Figure 2(b)) and occupy the $-5.89\left(\mathrm{~A}_{1}, 1\right),-3.31\left(\mathrm{~B}_{2}, 1\right)$, and $-2.92(\mathrm{E}, 2)$ orbitals. As a consequence, the two $\pi$-electron systems make the $\mathrm{AuGe}_{10}{ }^{-}$ and $\mathrm{AuGe}_{12}{ }^{-}$clusters spherically aromatic. This aromatic feature can be regarded as one of the main reasons in the structural stabilization of endohedrally doped $\mathrm{AuGe}_{10}{ }^{-}$ and $\mathrm{AuGe}_{12}{ }^{-}$clusters. However, it is noteworthy that the $2(N+1)^{2}$ electron counting rule cannot be solely used to explain the aromaticity of compounds. For instance, the bianionic $\mathrm{Si}_{12}{ }^{2-}$ cluster contains eight $\pi$ electrons, but gives 
an antiaromatic character [57]. Therefore, the aromaticity of both the $\mathrm{AuGe}_{10}{ }^{-}$and $\mathrm{AuGe}_{12}{ }^{-}$clusters needs to be further confirmed by NICS values given in next section.

3.4. NICS Values of Clusters. The aromaticity of a chemical compound can be usually discussed in terms of various criteria, but the criteria used to validate the aromaticity are sometimes controversial, likely $\mathrm{Si}_{12}{ }^{2-}$ cluster. Herein, we performed a calculation of NICS proposed by Chen and coworkers [33], based on magnetic shieldings. Aromaticity is expected to be evaluated by a negative NICS value, and antiaromaticity by a positive NICS value. In general, a ghost atom is placed at the center of a spherical geometry to obtain NICS value. In order to get a reasonable value, however, we considered many different sites along central axis inside or outside the cage to test the variation of NICS value, as depicted in Figure 1. A radius $\left(L_{\mathrm{ab}}\right)$ from the center site of the cage to each ghost atom is also defined with $0 \leq L_{\mathrm{ab}} \leq 3.0$.

Using the GIAO-B3LYP/LanL2DZ level of theory, we have calculated the NICS values of the $\mathrm{AuGe}_{10}{ }^{-}$and $\mathrm{AuGe}_{12}{ }^{-}$ clusters. In order to give a proper description of magnetic and electronic properties, a large basis set (cc-pVTZ-PP) in our NICS calculations is also necessary with the same method. The NICS values of the two clusters are given in Table 1. We see that the NICS value at the center site is the largest one among all considered sites, being -259.6 and -296.4 ppm for $\mathrm{AuGe}_{10}{ }^{-}$and $\mathrm{AuGe}_{12}{ }^{-}$clusters, respectively. From Figure 4 we see that the NICS values of the $\mathrm{AuGe}_{10}{ }^{-}$ cluster rapidly decreases to $L_{\mathrm{ab}}=1.5(-25.2 \mathrm{ppm})$ and then slowly decreases to $L_{\mathrm{ab}}=3.0(-8.5 \mathrm{ppm})$. Obviously, the NICS values close to the outer surface are relatively smaller than those inside the cage. Similar behavior is found for the $\mathrm{AuGe}_{12}{ }^{-}$cluster, and it gives a slightly larger NICS value than $\mathrm{AuGe}_{10}{ }^{-}$cluster. We also find that the NICS values with LanL2DZ basis set are in good agreement with the results with cc-pVTZ-PP basis set, except for the value at the center site that is relatively large with the deviation of about $20.0 \mathrm{ppm}$. Thus, the aromaticity of both the $\mathrm{AuGe}_{10}{ }^{-}$and $\mathrm{AuGe}_{12}{ }^{-}$clusters, characterized by the $2\left(N_{\pi}+1\right)^{2}\left[N_{\pi}=1\right]$ electron counting rule, is confirmed by largely negative NICS values. Moreover, the two clusters have very strong aromatic character at the center site, and the aromaticity will weaken outside the cage and vanish beyond.

\section{Conclusions}

Using the hybrid DFT-B3LYP functional, we have studied a large number of structural isomers for medium-sized $\mathrm{AuGe}_{10}{ }^{-}$and $\mathrm{AuGe}_{12}{ }^{-}$clusters. The endohedrally Au-doped pentagonal prism and bicapped pentagonal prism are found to be the most stable $\mathrm{AuGe}_{10}{ }^{-}\left(D_{5 h}\right)$ and $\mathrm{AuGe}_{12}{ }^{-}\left(D_{2 d}\right)$ structures, respectively. Analysis of reaction energy indicates that the endohedrally doped $\mathrm{AuGe}_{10}{ }^{-}$and $\mathrm{AuGe}_{12}{ }^{-}$clusters have the high thermodynamic stability. We see that the highenergy regions of molecular occupied orbitals of the two compounds come mainly from the contribution of $p$-Ge state by means of the partial density of states (PDOS). The electron localization functions (ELFs) are also considered

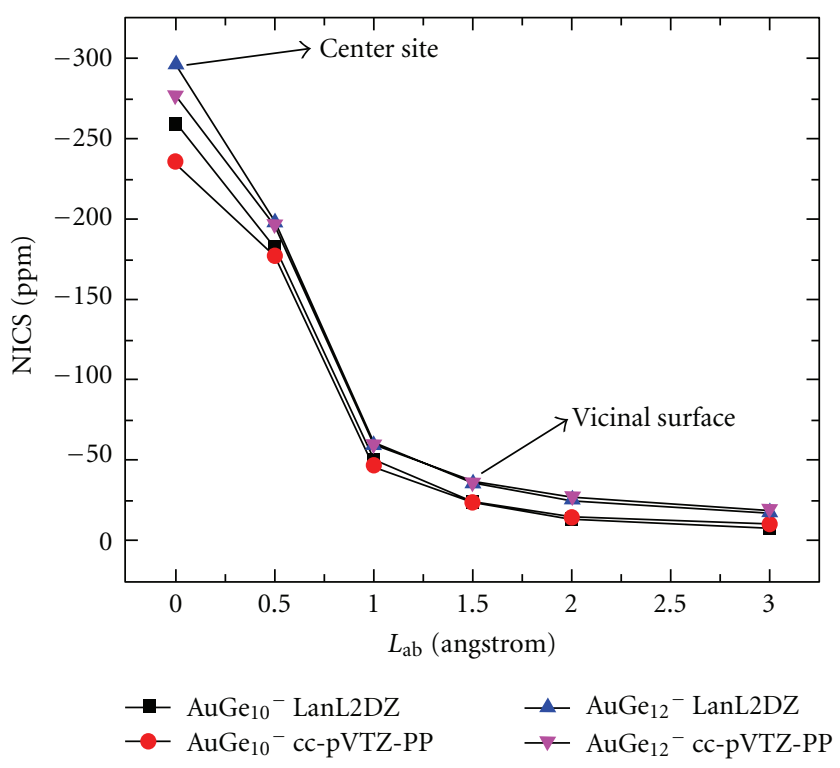

Figure 4: Comparison of NICS values of anionic $\mathrm{AuGe}_{10}{ }^{-}$, and $\mathrm{AuGe}_{12}{ }^{-}$clusters using two different LanL2DZ and cc-pVTZPP basis sets with the same B3LYP functional, based on the B3LYP/LanL2DZ geometries.

to explore the chemical bonding of the two clusters, and in some regions more metallic (or less covalent) character between germanium atoms becomes more preferable, for example, pentagonal prismy bonds. In the $\mathrm{AuGe}_{10}{ }^{-}$and AuGe ${ }_{12}{ }^{-}$structures, eight valence $\pi$-electrons satisfy the $2\left(N_{\pi}+1\right)^{2}\left[N_{\pi}=1\right]$ counting rule and make the $\mathrm{AuGe}_{10}{ }^{-}$ and $\mathrm{AuGe}_{12}{ }^{-}$clusters spherically aromatic. An NICS calculation on basis of magnetic shieldings is carried out to confirm the aromaticity of the three-dimensional clusters reflected by largely negative NICS values. Additionally, NICS is also relatively insensitive to the level of theory used in the study, for example, basis set.

\section{References}

[1] G. R. Burton, C. Xu, C. C. Arnold, and D. M. Neumark, "Photoelectron spectroscopy and zero electron kinetic energy spectroscopy of germanium cluster anions," The Journal of Chemical Physics, vol. 104, no. 8, pp. 2757-2764, 1996.

[2] M. Scheer, R. C. Bilodeau, C. A. Brodie, and H. K. Haugen, "Systematic study of the stable states of $\mathrm{C}^{-}, \mathrm{Si}^{-}, \mathrm{Ge}^{-}$, and $\mathrm{Sn}^{-}$ via infrared laser spectroscopy," Physical Review A, vol. 58, no. 4, pp. 2844-2856, 1998.

[3] Y. Negishi, H. Kawamata, T. Hayase et al., "Photoelectron spectroscopy of germanium-fluorine binary cluster anions: the HOMO-LUMO gap estimation of $\mathrm{Ge}_{n}$ clusters," Chemical Physics Letters, vol. 269, no. 3-4, pp. 199-207, 1997.

[4] J. Thøgersen, L. D. Steele, M. Scheer, C. A. Brodie, and H. K. Haugen, "Electron affinities of $\mathrm{Si}, \mathrm{Ge}, \mathrm{Sn}$ and Pt by tunable laser photodetachment studies," Journal of Physics B, vol. 29, no. 7, pp. 1323-1330, 1996.

[5] L.-Z. Zhao, W.-C. Lu, W. Qin, Q. J. Zang, C. Z. Wang, and K. M. Ho, "Fragmentation behavior of $\mathrm{Ge}_{n}$ clusters $(2<n<33)$," Chemical Physics Letters, vol. 455, no. 4-6, pp. 225-231, 2008. 
[6] D. Bandyopadhyay and P. Sen, "Density functional investigation of structure and stability of $\mathrm{Ge}_{n}$ and $\mathrm{Ge}_{n} \mathrm{Ni}(n=1-20)$ clusters: validity of the electron counting rule," Journal of Physical Chemistry A, vol. 114, no. 4, pp. 1835-1842, 2010.

[7] B. Yu, X. H. Sun, G. A. Calebotta, G. R. Dholakia, and M. Meyyappan, "One-dimensional germanium nanowires for future electronics," Journal of Cluster Science, vol. 17, no. 4, pp. 579-597, 2006.

[8] W.-J. Zhao and Y.-X. Wang, "Geometries, stabilities, and magnetic properties of $\mathrm{MnGe}_{n}(n=2-16)$ clusters: density-functional theory investigations," Journal of Molecular Structure, vol. 901, no. 1-3, pp. 18-23, 2009.

[9] W.-J. Zhao and Y.-X. Wang, "Geometries, stabilities, and electronic properties of $\mathrm{FeGe}_{n}(n=9-16)$ clusters: densityfunctional theory investigations," Chemical Physics, vol. 352, no. 1-3, pp. 291-296, 2008.

[10] V. Kumar and Y. Kawazoe, "Metal-encapsulated caged clusters of germanium with large gaps and different growth behavior than silicon," Physical Review Letters, vol. 88, no. 23, Article ID 235504, 4 pages, 2002.

[11] T. Van Hoof and M. Hou, "Structural and thermodynamic properties of Ag-Co nanoclusters," Physical Review B, vol. 72, no. 11, Article ID 115434, 12 pages, 2005.

[12] E. E. Zhurkin and M. Hou, "Structural and thermodynamic properties of elemental and bimetallic nanoclusters: an atomic scale study," Journal of Physics, vol. 12, no. 30, pp. 6735-6754, 2000.

[13] J. U. Reveles, P. Sen, K. Pradhan, D. R. Roy, and S. N. Khanna, "Effect of electronic and geometric shell closures on the stability of neutral and anionic $\mathrm{TiNa}_{n}(n=1-13)$ clusters," Journal of Physical Chemistry C, vol. 114, no. 24, pp. 1073910744, 2010.

[14] K. Meier, R. Cardoso-Gil, W. Schnelle, H. Rosner, U. Burkhardt, and U. Schwarz, "Thermal, magnetic, electronic, and superconducting properties of rare earth metal pentagermanides $\mathrm{REGe}_{5}(\mathrm{RE}=\mathrm{La}, \mathrm{Nd}, \mathrm{Sm}, \mathrm{Gd})$ and synthesis of $\mathrm{TbGe}_{5}$," Zeitschrift für Anorganische und Allgemeine Chemie, vol. 636, no. 8, pp. 1466-1473, 2010.

[15] S. Furuse, K. Koyasu, J. Atobe et al., "Experimental and theoretical characterization of $\mathrm{MSi}_{16}{ }^{-}, \mathrm{MGe}_{16}{ }^{-}, \mathrm{MSn}_{16}{ }^{-}$and $\mathrm{MPb}_{16}{ }^{-}(\mathrm{M}=\mathrm{Ti}, \mathrm{Zr}$, and $\mathrm{Hf})$ : the role of cage aromaticity," The Journal of Chemical Physics, vol. 129, no. 6, pp. 064311064316, 2008.

[16] X. Li and L.-S. Wang, "Experimental search and characterization of icosahedral clusters: $\mathrm{Al}_{12} X^{-}(X=\mathrm{C}, \mathrm{Ge}, \mathrm{Sn}, \mathrm{Pb})$," Physical Review B, vol. 65, no. 15, Article ID 153404, 4 pages, 2002.

[17] J. Wang and J.-G. Han, "A computational investigation of copper-doped germanium and germanium clusters by the density-functional theory," The Journal of Chemical Physics, vol. 123, no. 24, Article ID 244303, 12 pages, 2005.

[18] X.-J. Li and K.-H. Su, "Structure, stability and electronic property of the gold-doped germanium clusters: $\mathrm{AuGe}_{n}(n=$ 2 - 13)," Theoretical Chemistry Accounts, vol. 124, no. 5-6, pp. 345-354, 2009.

[19] V. Kumar and Y. Kawazoe, "Metal-encapsulated icosahedral superatoms of germanium and tin with large gaps: $\mathrm{ZnGe}_{12}$ and CdSn $n_{12}$," Applied Physics Letters, vol. 80, no. 5, pp. 859-861, 2002.

[20] J. Lu and S. Nagase, "Metal-doped germanium clusters $\mathrm{MGe}_{n} \mathrm{~s}$ at the sizes of $n=12$ and 10: divergence of growth patterns from the $\mathrm{MSi}_{n}$ clusters," Chemical Physics Letters, vol. 372, no. 3-4, pp. 394-398, 2003.
[21] X. Zhang, G. Li, and Z. Gao, "Laser ablation of Co/Ge mixtures: a new type of endohedral structure, a semiconductor cage trapping a metal atom," Rapid Communications in Mass Spectrometry, vol. 15, no. 17, pp. 1573-1576, 2001.

[22] Q. Jing, F.-Y. Tian, and Y.-X. Wang, "No quenching of magnetic moment for the $\mathrm{Ge}_{n} \mathrm{Co}(n=1-13)$ clusters: firstprinciples calculations," The Journal of Chemical Physics, vol. 128, no. 12, Article ID 124319, 5 pages, 2008.

[23] J. Wang and J.-G. Han, "A theoretical study on growth patterns of Ni-doped germanium clusters," Journal of Physical Chemistry B, vol. 110, no. 15, pp. 7820-7827, 2006.

[24] N. Kapila, I. Garg, V. K. Jindal et al., "First principle investigation into structural growth and magnetic properties in $\mathrm{Ge}_{n} \mathrm{Cr}$ clusters for $n=1-13$," Journal of Magnetism and Magnetic Materials, vol. 324, no. 18, pp. 2885-2893, 2012.

[25] J. Wang and J.-G. Han, "Geometries and electronic properties of the tungsten-doped germanium clusters: $\mathrm{WGe}_{n}(n=1-17$ )," Journal of Physical Chemistry A, vol. 110, no. 46, pp. 1267012677, 2006.

[26] M. Kumar, N. Bhattacharyya, and D. Bandyopadhyay, "Architecture, electronic structure and stability of TM@Ge ( TM $=\mathrm{Ti}, \mathrm{Zr}$ and Hf, $n=1-20)$ clusters: a density functional modeling," Journal of Molecular Modeling, vol. 18, no. 1, pp. 405-418, 2012.

[27] D. Bandyopadhyay, "Architectures, electronic structures, and stabilities of $\mathrm{Cu}$-doped $\mathrm{Ge}_{n}$ clusters: density functional modeling," Journal of Molecular Modeling, vol. 18, no. 8, pp. 38873902, 2012.

[28] V. Kumar, A. K. Singh, and Y. Kawazoe, "Smallest magic caged clusters of $\mathrm{Si}, \mathrm{Ge}, \mathrm{Sn}$, and $\mathrm{Pb}$ by encapsulation of transition metal atom," Nano Letters, vol. 4, no. 4, pp. 677-681, 2004.

[29] M. M. Uță, D. Cioloboc, and R. B. King, "Cobalt-centered ten-vertex germanium clusters: the pentagonal prism as an alternative to polyhedra predicted by the wade-mingos rules," Inorganic Chemistry, vol. 51, no. 6, pp. 3498-3504, 2012.

[30] R. B. King, I. Silaghi-Dumitrescu, and M. M. Uțǎ, "Endohedral beryllium atoms in ten-vertex germanium clusters: effect of a small interstitial atom on the cluster geometry," Journal of Physical Chemistry A, vol. 115, no. 13, pp. 2847-2852, 2011.

[31] C. Tang, M. Liu, W. Zhu et al., "Probing the geometric, optical, and magnetic properties of $3 \mathrm{~d}$ transition-metal endohedral $\mathrm{Ge}_{12} \mathrm{M}(\mathrm{M}=\mathrm{Sc}-\mathrm{Ni})$ clusters," Computational and Theoretical Chemistry, vol. 969, no. 1-3, pp. 56-60, 2011.

[32] A. Hirsch, Z. Chen, and H. Jiao, "Spherical aromaticity in $I_{\mathrm{h}}$ symmetrical fullerenes: the $2(N+1)^{2}$ rule," Angewandte Chemie, vol. 39, no. 21, pp. 3915-3917, 2000.

[33] Z. Chen, C. S. Wannere, C. Corminboeuf, R. Puchta, and P. von Ragué Schleyer, "Nucleus-independent chemical shifts (NICS) as an aromaticity criterion," Chemical Reviews, vol. 105 , no. 10, pp. 3842-3888, 2005.

[34] A. I. Boldyrev and L.-S. Wang, "All-metal aromaticity and antiaromaticity," Chemical Reviews, vol. 105, no. 10, pp. 37163757, 2005.

[35] L.-M. Wang, S. Bulusu, W. Huang, R. Pal, L. S. Wang, and C. Z. Xiao, "Doping the golden cage $\mathrm{Au}_{16}{ }^{-}$with $\mathrm{Si}, \mathrm{Ge}$, and $\mathrm{Sn}$," Journal of the American Chemical Society, vol. 129, no. 49, pp. 15136-15137, 2007.

[36] R. Pal, L.-M. Wang, W. Huang, L. S. Wang, and X. C. Zeng, "Structural evolution of doped gold clusters: $\mathrm{MAu}_{x}{ }^{-}(\mathrm{M}=\mathrm{Si}$, Ge, Sn; $x=5-8)$," Journal of the American Chemical Society, vol. 131, no. 9, pp. 3396-3404, 2009.

[37] M. Akutsu, K. Koyasu, J. Atobe, K. Miyajima, M. Mitsui, and A. Nakajima, "Electronic properties of Si and Ge atoms doped 
in clusters: $\operatorname{In}_{n} \mathrm{Si}_{m}$ and $\operatorname{In}_{n} \mathrm{Ge}_{m}$," Journal of Physical Chemistry A, vol. 111, no. 4, pp. 573-577, 2007.

[38] C. Lee, W. Yang, and R. G. Parr, "Development of the ColleSalvetti correlation-energy formula into a functional of the electron density," Physical Review B, vol. 37, no. 2, pp. 785$789,1988$.

[39] A. D. Becke, "Density-functional exchange-energy approximation with correct asymptotic behavior," Physical Review A, vol. 38, no. 6, pp. 3098-3100, 1988.

[40] M. J. Frisch, G. W. Trucks, H. B. Schlegel et al., Gaussian 09, Revision A. 02, Gaussian, Inc., Wallingford, UK, 2009.

[41] P. J. Hay and W. R. Wadt, "Ab initio effective core potentials for molecular calculations. Potentials for the transition metal atoms Sc to Hg," The Journal of Chemical Physics, vol. 82, no. 1, pp. 270-283, 1985.

[42] W. R. Wadt and P. J. Hay, "Ab initio effective core potentials for molecular calculations. Potentials for main group elements $\mathrm{Na}$ to Bi," The Journal of Chemical Physics, vol. 82, no. 1, pp. 284298, 1985.

[43] P. J. Hay and W. R. Wadt, "Ab initio effective core potentials for molecular calculations. Potentials for $\mathrm{K}$ to Au including the outermost core orbitale," The Journal of Chemical Physics, vol. 82, no. 1, pp. 299-310, 1985.

[44] X.-J. Li, K.-H. Su, X.-H. Yang et al., "Size-selective effects of geometry and electronic property on bimetallic Au-Ge nanoclusters," to appear in. Computational and Theoretical Chemistry.

[45] N. M. O’Boyle, A. L. Tenderholt, and K. M. Langner, "Cclib: a library for package-independent computational chemistry algorithms," Journal of Computational Chemistry, vol. 29, no. 5, pp. 839-845, 2008.

[46] F. London, "Théorie quantique des courants interatomiques dans les combinaisons aromatiques," Journal de Physique et Le Radium, vol. 8, no. 10, pp. 397-409, 1937.

[47] G. Schreckenbach and T. Ziegler, "Density functional calculations of NMR chemical shifts and ESR g-tensors," Theoretical Chemistry Accounts, vol. 99, no. 2, pp. 71-82, 1998.

[48] A. K. Wilson, D. E. Woon, K. A. Peterson, and T. H. Dunning, "Gaussian basis sets for use in correlated molecular calculations. IX. The atoms gallium through krypton," The Journal of Chemical Physics, vol. 110, no. 16, pp. 7667-7676, 1999.

[49] D. Figgen, G. Rauhut, M. Dolg, and H. Stoll, "Energyconsistent pseudopotentials for group 11 and 12 atoms: adjustment to multi-configuration Dirac-Hartree-Fock data," Chemical Physics, vol. 311, no. 1-2, pp. 227-244, 2005.

[50] J. Wang and J. G. Han, "The growth behaviors of the Zndoped different sized germanium clusters: a density functional investigation," Chemical Physics, vol. 342, no. 1-3, pp. 253259, 2007.

[51] J. E. Huheey, E. A. Keiter, and R. L. Keiter, Inorganic Chemistry: Principles of Structure and Reactivity, Harper Collins College, New York, NY, USA, 4th edition, 2000.

[52] P. Sen and L. Mitas, "Electronic structure and ground states of transition metals encapsulated in a $\mathrm{Si}_{12}$ hexagonal prism cage," Physical Review B, vol. 68, no. 15, Article ID 155404, 4 pages, 2003.

[53] B. Silvi and A. Savin, "Classification of chemical bonds based on topological analysis of electron localization functions," Nature, vol. 371, no. 6499, pp. 683-686, 1994.

[54] K. Joshi, D. G. Kanhere, and S. A. Blundell, "Abnormally high melting temperature of the $\mathrm{Sn}_{10}$ cluster," Physical Review B, vol. 66, no. 15, Article ID 155329, 5 pages, 2002.
[55] A. Hirsch, Z. Chen, and H. Jiao, "Spherical aromaticity of inorganic cage molecules," Angewandte Chemie, vol. 40, no. 15, pp. 2834-2838, 2001.

[56] T. B. Tai and M. T. Nguyen, "Lithium atom can be doped at the center of a germanium cage: the stable icosahedral $\mathrm{Ge}_{12} \mathrm{Li}^{-}$ cluster and derivatives," Chemical Physics Letters, vol. 492, no. 4-6, pp. 290-296, 2010.

[57] R. B. King, T. Heine, C. Corminboeuf, and P. V. R. Schleyer, "Antiaromaticity in bare deltahedral silicon clusters satisfying Wade's and Hirsch's rules: an apparent correlation of antiaromaticity with high symmetry," Journal of the American Chemical Society, vol. 126, no. 2, pp. 430-431, 2004. 

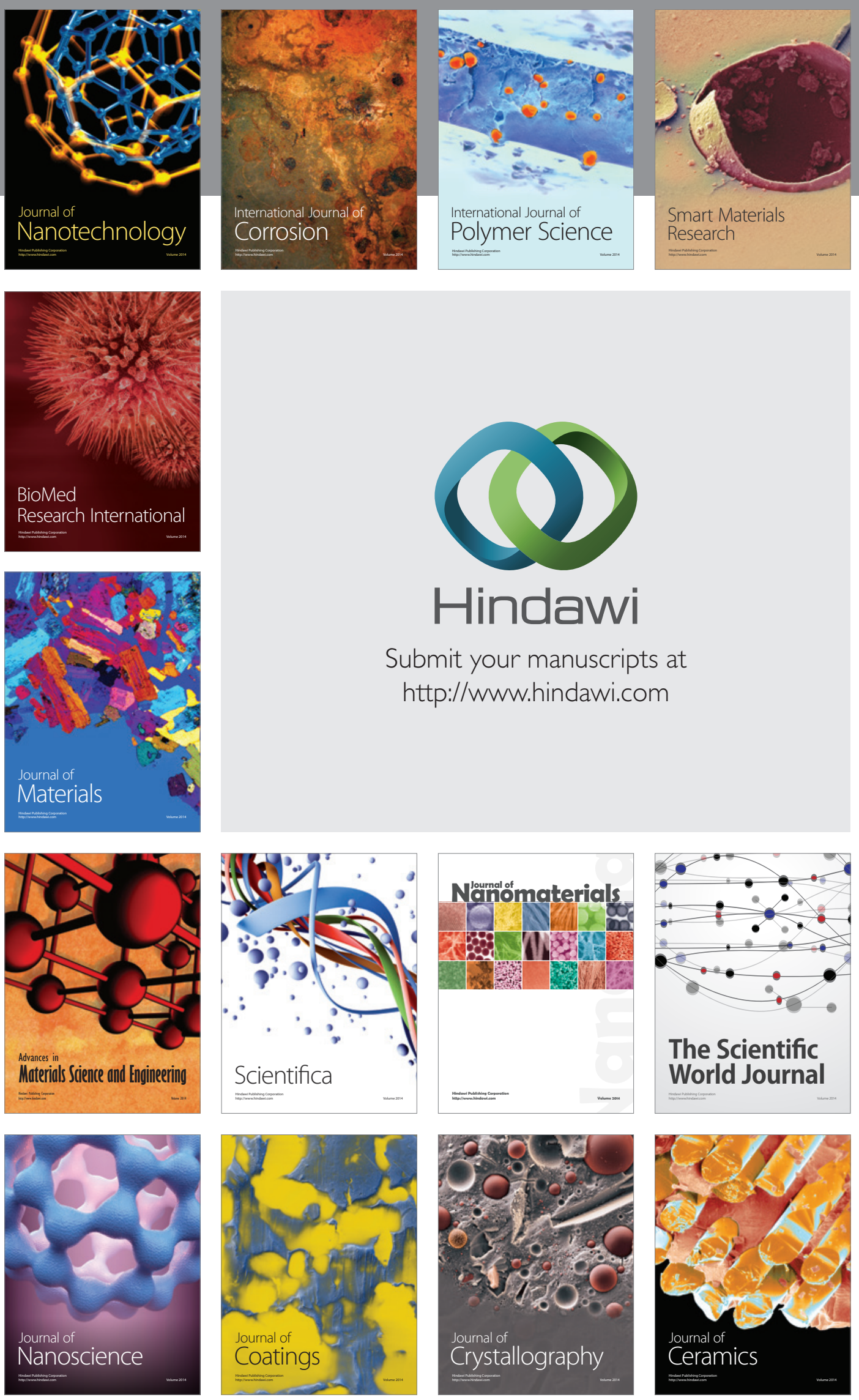

The Scientific World Journal

Submit your manuscripts at

http://www.hindawi.com

\section{World Journal}

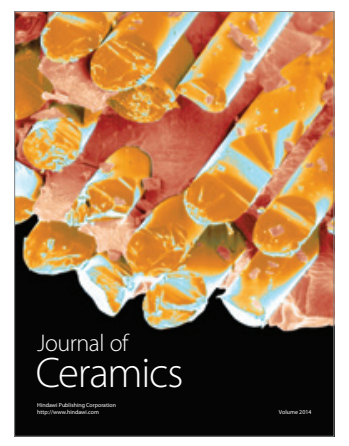

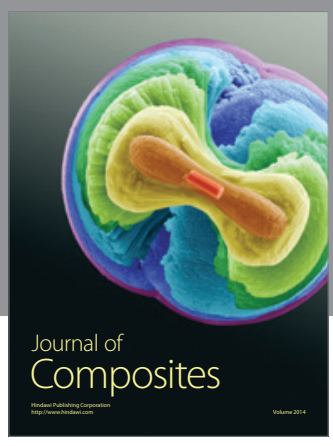
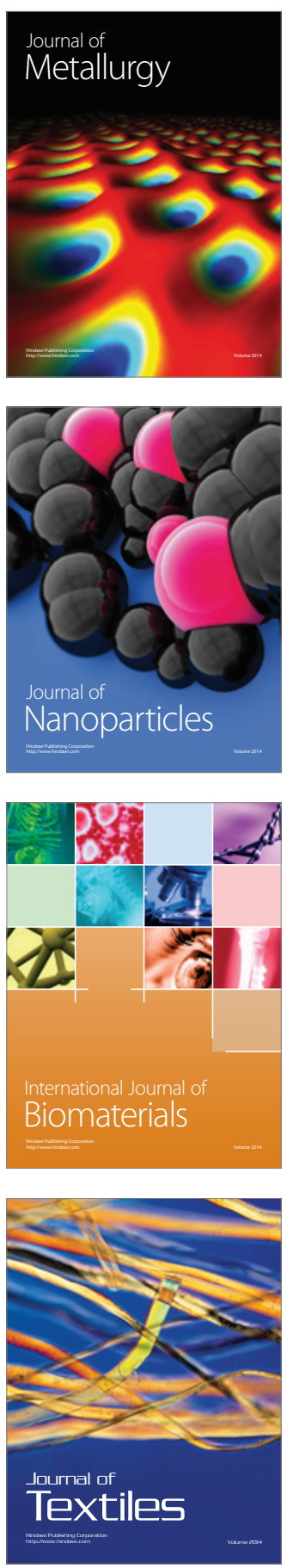